\title{
Transform Domain Fingerprint Identification Based on DTCWT
}

\author{
Jossy P. George \\ Department of Computer Science \\ Christ University \\ Bangalore, Karnataka, India
}

\author{
Abhilash S. K., Raja K. B. \\ Dept. of Electronics and Communication Engineering \\ University Visvesvaraya College of Engineering \\ Bangalore, Karnataka, India
}

\begin{abstract}
The physiological biometric characteristics are better compared to behavioral biometric identification of human beings to identify a person. In this paper, we propose Transform Domain Fingerprint Identification Based on DTCWT. The original Fingerprint is cropped and resized to suitable dimension to apply DTCWT. The DTCWT is applied on Fingerprint to generate coefficient which form features. The performance analysis is discussed with different levels of DTCWT and also with different sizes of Fingerprint database. It is observed that the recognition rate is better in the case of level 7 compared to other levels of DTCWT.
\end{abstract}

Keywords-Fingerprint; DTCWT; Euclidean Distance; Preprocessing

\section{INTRODUCTION}

The term biometric is derived from the Greek word bio (life) and metrics (to measure). The biometrics identifies the person, based on feature vectors derived from physiological or behavioral characteristics. The biometric traits must satisfy universality, uniqueness, permanence, accessibility, collectability. The physiological biometrics are Fingerprint, Hand Scan, Iris Scan, Facial Scan and Retina Scan etc. and behavioral bio-metric are Voice, Keystroke, Gait, Signature etc. The physiological biometrics traits are almost constant throughout the life span of a person [1], even for identical twins. The behavior biometric trait varies with mood and environment.

The bio-metric authentication is better than traditional methods. The existing traditional methods to authenticate are Passwords, Personnel Identification Numbers (PINs), Tokens and Smart Cards. The disadvantages of traditional methods are (i) Passwords and Pins are difficult to remember. (ii) More chances of losing tokens and smart cards. (iii) The misuse of traditional methods of authentication by miscreants is very high, especially in the case of money transaction through ATMs, access to the unauthorized places, etc.

Biometric traits are identified in different domains, such as spatial, transformation and hybrid domain. In spatial domain biometric traits are considered as image itself, wherein features of image are obtained by computing the area, height, width, pixel density, mean, variance and standard deviation etc. In transform domain, the spatial domain image is converted into other domain using Fast Fourier Transform (FFT), The Discrete Cosine Transform (DCT), Discrete Wavelet Transform (DWT), Dual-Tree Complex Wavelet Transform (DTCWT), Empirical Mode Decomposition (EMD), Singular Value Decomposition (SVD), Principal Components Analysis
(PCA), Independent Component Analysis (ICA), etc. These coefficients of transform domain form feature vectors. In hybrid domain, the combination of spatial and transformation domain features are obtained. The features of spatial, transformation and hybrid domains are compared using distance vector formulae such as Equivalent Distance (ED), Hamming Distance (HD), Chi-square test, or classifiers such as Neural Networks (NN), Support Vector Machine (SVM) and Random Forest (RF).

Fingerprint is a physiological trait which is constant throughout the life span of a person. The fingerprint is unique even for the twins. It has been used as the identification technique for over a century. The fingerprint authentication officially established as a means of identifying people around 1900s. This authentication got the popularity because; the devices for accessing the fingerprints are small and inexpensive. When a biometric verification is to occur, a scan of the biometric of a person is made and which is to be compared with the stored data of the same person.

A fingerprint usually appears as a series of dark lines that represents the high, peaking portion of the friction ridge skin, while the valleys between these ridges appears as white space and are the low, shallow portion of the friction ridge skin, minutiae, or the location and direction of the ridge endings and bifurcations along a ridge path. The upper most point on the inner most ridge of the fingerprint image is known as core. The fingerprints are mainly classified as Arch, Tented Arch, Whorl, Left Loop, and Right Loop. There are separating point between pattern area and non-pattern area in many of the fingerprint images. These points are generally known as delta.

A. Contribution: In this paper the Transform Domain Fingerprint Identification Based on DTCWT at different levels is introduced. DTCWT generates complex coefficients by using a dual tree of wavelet filters to obtain two parts of the images, i.e., real and imaginary part. DTCWT is applied on Fingerprint to generate different levels to obtain Fingerprint features.

B. Organization: The Introduction is given in section I, the existing research papers are discussed in section II, the proposed model is explained in section III, the algorithm is described in section IV, the performance analysis is discussed in section $\mathrm{V}$ and finally, conclusion is given in section VI. 


\section{LITERATURE SURVEY}

The Fingerprint for individual identification and verification with existing different technique and applications are described in this section.

S. Vasuki et. al., [2] proposed a model for segmentation of a color textured regions of a given images. This is obtained by the segmentation and by applying DTCWT. This model works in two levels where in first level, after applying DTCWT, the image is divided into 16 sub images and from where the maximum energy sub image is selected as an optimum feature space. In the second level, K-means spatial refinement algorithm is applied. The main advantage of this model is the accuracy. Zhao Song and Liu Yuanpeng [3] gave a novel image diagnosing scheme by applying 2-D DTCWT to the second bandlet transform. This is obtained based on the shift invariance and better directionality of the DTCWT. The bandlet reconstruction recovers the transitions and directional textures. This improves significantly image diagnosing results. Chen Feng and Yu Song - nian [4] proposed a model which retrieves the multi scale image by using a new class of image features as the image descriptors from DTCWT. From this work, we can conclude that the performance of DTCWT is better in the experiment on the stander COREL image database due to the rotation invariance, translation invariance, robust to noise and getting the key point according to people's cognitive habits. Sathesh and Samuel Manoharan [5] discussed on advantages and disadvantages of DWT. Also they give the methods to overcome the limitations of DWT and the theoretical analysis of complex wavelet transform and its verification using the simulated images. V. Lulian and B. Monica [6] proposed a method to design and optimize separately two channels perfect reconstruction filter banks. This method ensures the good quality for the two levels. This method is more useful where the only the few levels of decomposition is required.

Shahid and Gupta S [7] proposed a novel method to fuse an image using the DTCWT. This is achieved through the formation of a fused pyramid using DTCWT coefficients from the decomposed pyramids of a source image. This method gives better qualitative and quantitative results than the DWT methods. Yun and Cho [8] proposed an adaptive preprocessing method, which extracts five features from the fingerprint images, analyzes image quality with clustering method, and enhances the images according to their characteristics. The preprocessing is performed after distinguishing the fingerprint image quality according to its characteristics. Brankica M. Popovi'c and L. Maskovic [9] used multi scale directional information obtained from orientation field image to filter the spurious minutiae. The feature extraction in pattern recognition system is to extract information from the input data and depends greatly on the quality of the images. Multi scale directional information estimated based on orientation field estimation. F. A. Afar et. al., [10] presented the minutiae based Automatic Fingerprint Identification Systems. The technique is based on the extraction of minutiae from the thinned, binarized and segmented version of a fingerprint image. The system uses fingerprint classification for indexing during fingerprint matching. G. Jagadeeswar Reddy et. al., [11] presented fingerprint diagnosing using both wavelet and Curvelet
Transforms. The search-rearrangement method performs better than minutiae based matching for fingerprint binary constraint graph matching since it does not require implicit alignment of two fingerprint images.

K. Zebbiche and F. Khelifi [12] presented biometric images as one Region of Interest (ROI) that has the data processed by most biometric based system. The scheme consists of embedding the watermark into ROI in fingerprint images. Discrete Wavelet Transform and Discrete Fourier Transform are used. Bhupesh Gour et. al., [13] introduced midpoint ridge contour representation in order to extract the minutiae from fingerprint images. Color coding scheme is used to scan each ridge only once. Seung Hoon chae and Jong Ku Kim [14] proposed Fingerprint Verification in which both minutiae and ridge information are used to reduce the errors due to incomplete alignment or distortion. This works gives more importance to the areas where we get errors in processing the algorithms. Aparecido Nilcau Marana and A. K. Jain [15] proposed Ridge Based Fingerprint matching using the Hough transform. The major straight lines that match the fingerprint ridges are used to estimate rotation and translation parameters. This method gives better and accurate results. Anil K Jain et al., [16] described the use of logistic regression method to integrate multiple fingerprint matching algorithms. The integration of Hough transform based matching, string distance based matching and 2D dynamic programming based matching using the logistic regression has minimized the False Rejection Rate for a specified level of False Acceptance Ratio.

Fanglin Chen and Jie Zhou [17] proposed an algorithm for reconstructing fingerprint orientation field from saved minutiae and are used in the matching stage to compare with the minutiae from the query fingerprint. The orientation fields computed from the saved minutiae is a global feature and the saved minutia is the local feature, are used to get more information. Chunxian Ren and Yilong Yin [18] used the hybrid algorithm based on linear classifier to segregate foreground and background blocks. The pixel wise classifier uses three pixel features such as Coherence, mean and variance. Hartwig Front haler and Klaus Kollreider [19] used a multi grid representation of a discrete differential scale space enhancement strategy of fingerprint recognition system. The fingerprint image is decomposed using Laplacian Pyramid as relevant information is concentrated within a few frequency bands. The Fausian Directional Filtering is used to enhance ridge valley pattern of fingerprint using 1-D filtering on higher pyramid level. The linear symmetric features are used to extract the local ridge -valley orientation. D. R. Shashi Kumar et. al., [20] proposed fingerprint verification based on fusion of minutiae and ridges using strength factors. In this model, to extract minutiae and ridges, block filter and Hough Transform are used. This proposed algorithm gives better results than the many other existing ones. This is achieved by fusing the minutiae and the ridge parameters using strength factors.

\section{MODEL}

In this section, definitions of Performance Analysis and proposed model are discussed. 


\section{A. Definitons}

a) False Accept Rate (FAR): It is the probability that system incorrectly matches with images stored with input image database. The FAR can be calculated using Equation 1.

FAR $=\frac{\text { No. of persons accepted from out of database }}{\text { Total no. of persons in database. }}$

b) False Rejection Rate (FRR): It is the probability that system fails to recognize the correct pattern to match with the database images. It is the ratio of number of correct persons rejected in the database to the total number of persons in database and can be calculated using Equation 2 .

FRR $=\frac{\text { No. of correct persons rejected }}{\text { Total no. of persons in database. }}$

c) Equal Error Rate (EER): It is the value where both the reject and accept rates are equal.

d) True Success Rate (TSR): It is the ratio of total number of persons correctly matched in the database to the total number of persons in the database and is given by Equation 3.

$\mathrm{TSR}=$ No. of persons correctly Matched in the database Total no. of persons in database.

\section{B. Proposed Model}

The proposed model of Fingerprint Identification using DTCWT is given in the Fig. 1.

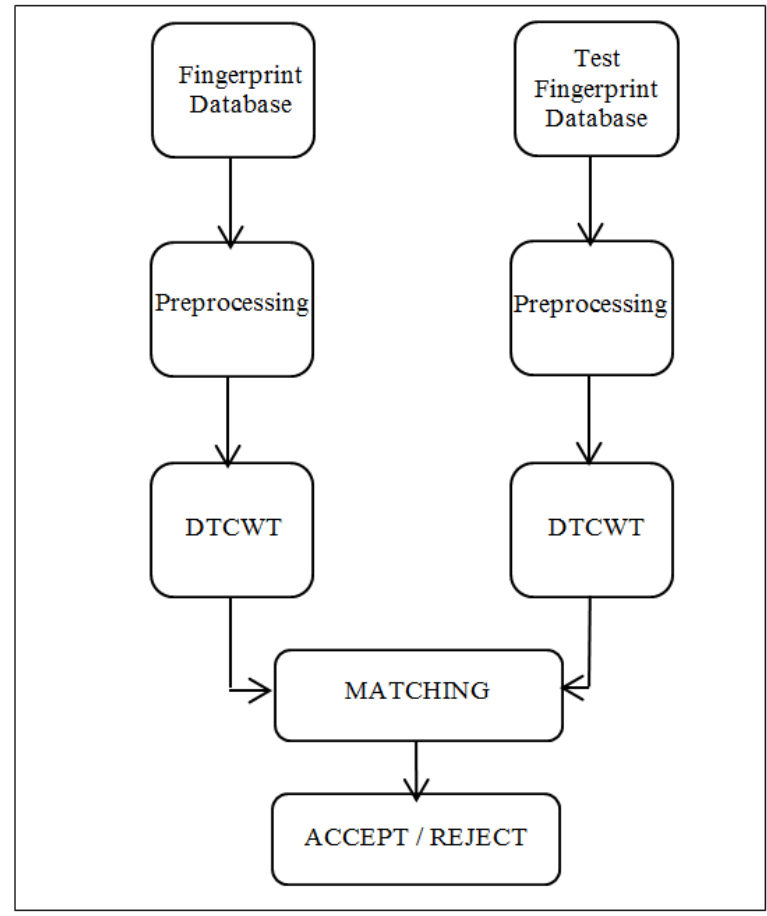

Figure 1. Proposed model

a) Fingerprint Database: Database collection is one of the important works in testing the biometric system. The best and advisable way of collecting the database is with a different sensor. There are different databases made available for the researchers to study on the different biometrics systems. The Fingerprint database available are the first, second and third International Competition on Fingerprint verification such as FVC 2000, FVC 2002 and FVC 2004 [21] respectively.

Four distinct databases for FVC 2004 provided by the organizers constitute the benchmark: DB1, DB2, DB3 and DB4. Each database is 110 fingers wide and 8 samples per finger in depth i.e., it consists of 880 fingerprint images. Each database is partitioned into disjoint subsets $\mathrm{A}$ and $\mathrm{B}$.

The subsets DB1-A, DB2-A, DB3-A and DB4-A, which contain the first 100 fingers (800 images) of DB1, DB2, DB3 and DB4, respectively, is used for the algorithm performance evaluation. The subsets DB1-B, DB2-B, DB3-B and DB4-B, containing the last 10 fingers (80 images) of DB1, DB2, DB3 and DB4 respectively made available to the participants to allow parameter tuning before executable(s) submission.

The Fig. 2 shows a sample image from each database. The DB3_A database is considered to test our algorithm due to its high resolution and size compatibility. A sample of finger print with eight impressions of DB3 is given in Fig. 3.

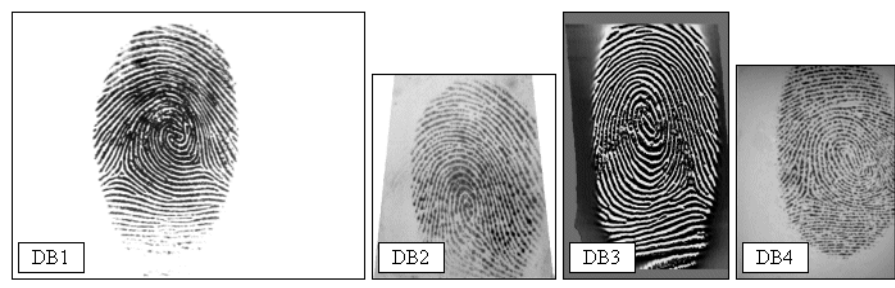

Figure 2. One fingerprint image from each database
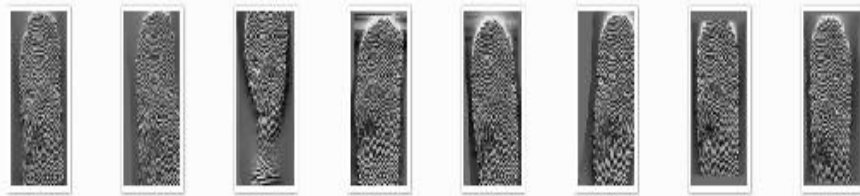

Figure 3. Fingerprint sample of DB3_A

- $\quad$ Source Database: The first seven Fingerprint images of each person from DB3 _A database of FVC 2004 are stored.

- Test Template: The eighth Fingerprint of each person from DB3 _A database of FVC 2004 are used in the test template and is compared with source database to compute FRR and TSR.

- Mismatch template database: The DB3_B of FVC 2004 database of 10 fingers are stored in Mismatch template database and compared with source database to compute FAR.

b) Pre-processing: The original Fingerprint image is of size 480 X 300. An observing the DB3_A of FVC 2004, we crop the input image to the size 401 X 201 in order to remove the unwanted portion in the image. And then the cropped image is resized into $512 \times 256$ for the DTCWT requirement. 
c) DTCWT: Dual Tree Complex Wavelet Transform is a recent enhancement technique to the Discrete Wavelet Transform with some additional properties and changes. It is a effective method for implementing an analytical wavelet transform, introduced by Kings bury in 1998 [22], [23], [24].
DTCWT gives the complex transform of a signal using two separate DWT decompositions ie., tree ' $a$ ' and tree ' $b$ '. DTCWT produces complex coefficients by using a dual tree of wavelet filters and gives real and imaginary parts which are shown in Fig 4.

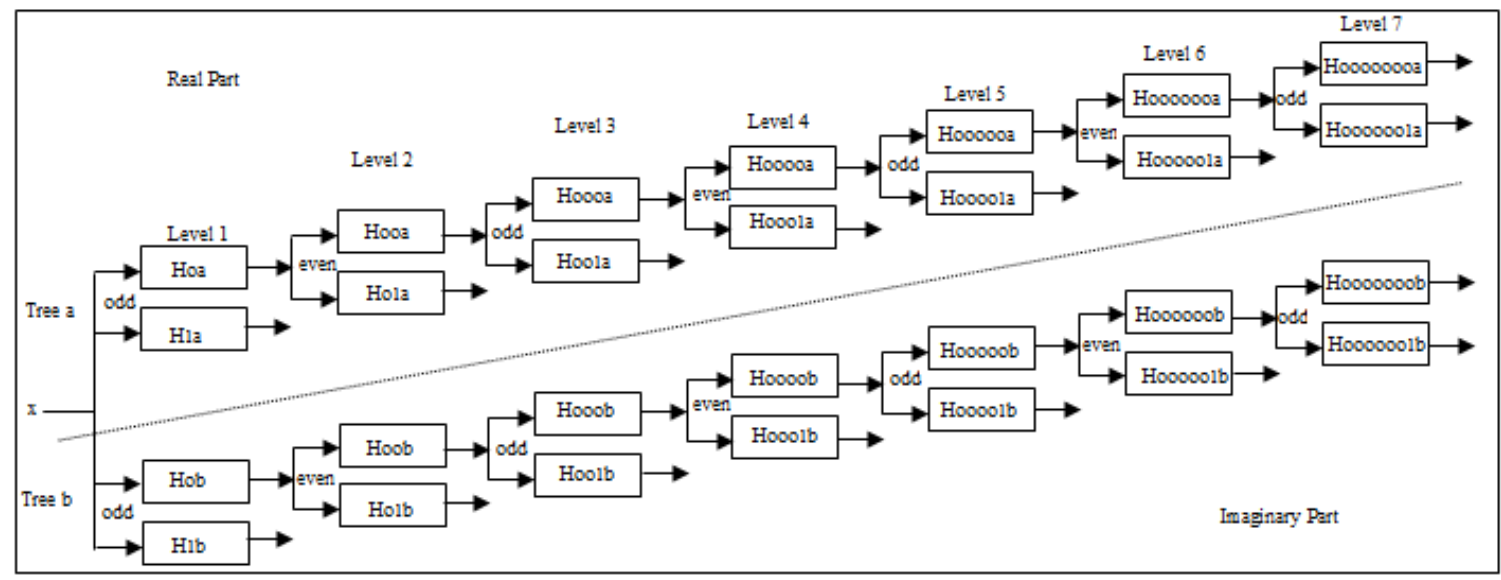

Figure 4. Real and imaginary parts of the complex coefficients

The DTCWT has following properties:

- Approximate shift invariance;

- Good directional selectivity in 2-dimensions (2-D) with Gabor-like filters also true for higher dimensionality: m-D);

- Perfect reconstruction (PR) using short linear-phase filters;

- Limited redundancy: independent of the number of scales: $2: 1$ for $1-\mathrm{D}$ ( $2 \mathrm{~m}: 1$ for $\mathrm{m}-\mathrm{D})$;

- Efficient order-N computation - only.

- DTCWT differentiates positive and negative frequencies and generates six sub bands oriented in $\pm 15^{\circ}, \pm 45^{\circ}$, and $\pm 75^{\circ}$. The different levels of DTCWT such as levels 5, 6, and 7 are applied on pre-processed Fingerprint Image.

d) Matching: The Euclidian Distance (ED) is used to verify the test image with the database images using the Equation 4.

$$
d 1(p, q)=\sqrt{\frac{1}{M} \sum_{i=1}^{M}\left(p_{i}-q_{i}\right)^{2}}
$$

Where,

$M=$ the dimension of feature vector.

$\mathrm{Pi}=$ is the database feature vector.

$\mathrm{qi}=$ is the test feature vector.

\section{ALGORITHM}

\section{A. Problem Definition}

The physiological trait Fingerprint is used to identify a person using the features obtained by the coefficients of DTCWT. The proposed algorithm for the performance analysis of the fingerprint identification for different levels of DTCWT is given in the Table1.

The objectives are;

- Fingerprint verification to authenticate a person using DTCWT

- To achieve high TSR

- To have FRR and FAR very low

While applying the DTCWT in different levels, the number of features and dimensions are reduces. The Fingerprint images for level-1, level-2, level-3, level-4 and level-5, level-6, level-7 are shown in Fig. 5.

TABLE 1. PROPOSED ALGORITHM.

1) FVC 2004, DB3_A database is considered.

2) Pre-processing is done by cropping the input fingerprint image to $401 \times 201$.

3) Cropped image is resized to 512 X 256 for DTCWT requirement.

4) DTCWT is applied on Fingerprint with levels 5, 6 and 7.

5) Magnitude and phase resulted from DTCWT are concatenated and considered as features.

6) Source database is created with the features obtained by step 5 .

7) For the test fingerprint DTCWT is applied and features obtained using step 5.

8) Test Fingerprint is compared with the database fingerprint using $E D$ to verify a person 


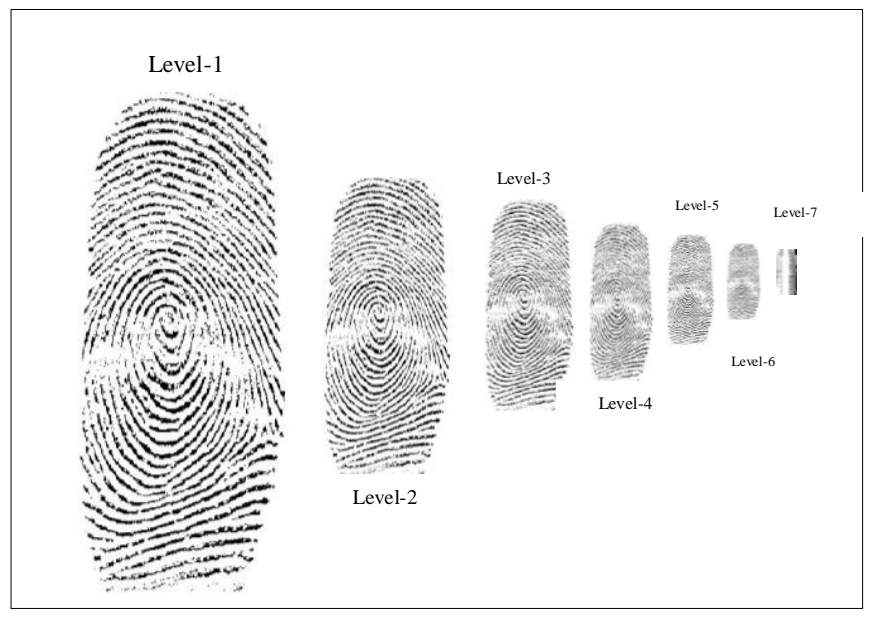

Figure 5. DTCWT images at different levels.

\section{PERFORMANCE ANALYSIS}

For the performance analysis, DB3_A of FVC 2004 Fingerprint database is considered. The number of persons inside the database (PIDB) to compute FRR and TSR are varied from 30 to 90 and the number of persons outside the database (PODB) are 10 to compute FAR is given in Table 2.

It is observed from the table 2 that the values of EER and TSR depend on the quality of Fingerprint image than the number of images in PIDB and PODB. The values of EER and TSR are better in the case of PIDB: PODB of 40:10. The performance of recognition rate is better in DTCWT level 7 compared to other lower levels of DTCWT. The TSR and EER is $85 \%$ and 0.15 respectively for DTCWT level 7 with PIDB: PODB of 40:10.

The graph for FRR, FAR and TSR is given in Fig. 6 and the variations of FRR and TSR with threshold for POIB: PODB of 40:10 is tabulated in Table 3 and It is noticed that as threshold increases, the value of FRR decreases, whereas the values of FAR and TSR increases. The highest success rate of recognition of $85 \%$ is achieved for the threshold value of 2.4 .

\section{CONCLUSION}

The Fingerprint biometric is used to authenticate a person. In this paper, Transform Domain Fingerprint Identification Based on DTCWT is proposed. The Fingerprint is preprocessed to a suitable size that suit DTCWT. The Fingerprint features are obtained by applying DTCWT with different levels. The test image features are compared with Database images using Euclidean Distance. It is observed that the recognition rate is better in the case of DTCWT level 7 compared to lower levels with PIDB: PODB of 40:10. In future, the algorithm may be combined with spatial domain features such as global and local features to enhance recognition rate.

\begin{tabular}{|c|c|c|c|c|c|c|c|}
\hline \multirow{2}{*}{ levels } & & \multicolumn{6}{|c|}{ PIDB:PODB } \\
\hline & & $30: 10$ & $40: 10$ & $60: 10$ & $70: 10$ & $80: 10$ & $90: 10$ \\
\hline \multirow{2}{*}{5} & EER & 0.5 & 0.2 & 0.573 & 0.34 & 0.36 & 0.33 \\
\hline & TSR & $50 \%$ & $80 \%$ & $42.7 \%$ & $66 \%$ & $64 \%$ & $67 \%$ \\
\hline \multirow{2}{*}{6} & EER & 0.45 & 0.2 & 0.59 & 0.3 & 0.282 & 0.3 \\
\hline & TSR & $55 \%$ & $80 \%$ & $41 \%$ & $70 \%$ & $71.8 \%$ & $70 \%$ \\
\hline \multirow{2}{*}{7} & EER & 0.36 & 0.15 & 0.228 & 0.21 & 0.197 & 0.197 \\
\hline & TSR & $64 \%$ & $85 \%$ & $77.2 \%$ & $79 \%$ & $80.3 \%$ & $82.1 \%$ \\
\hline
\end{tabular}

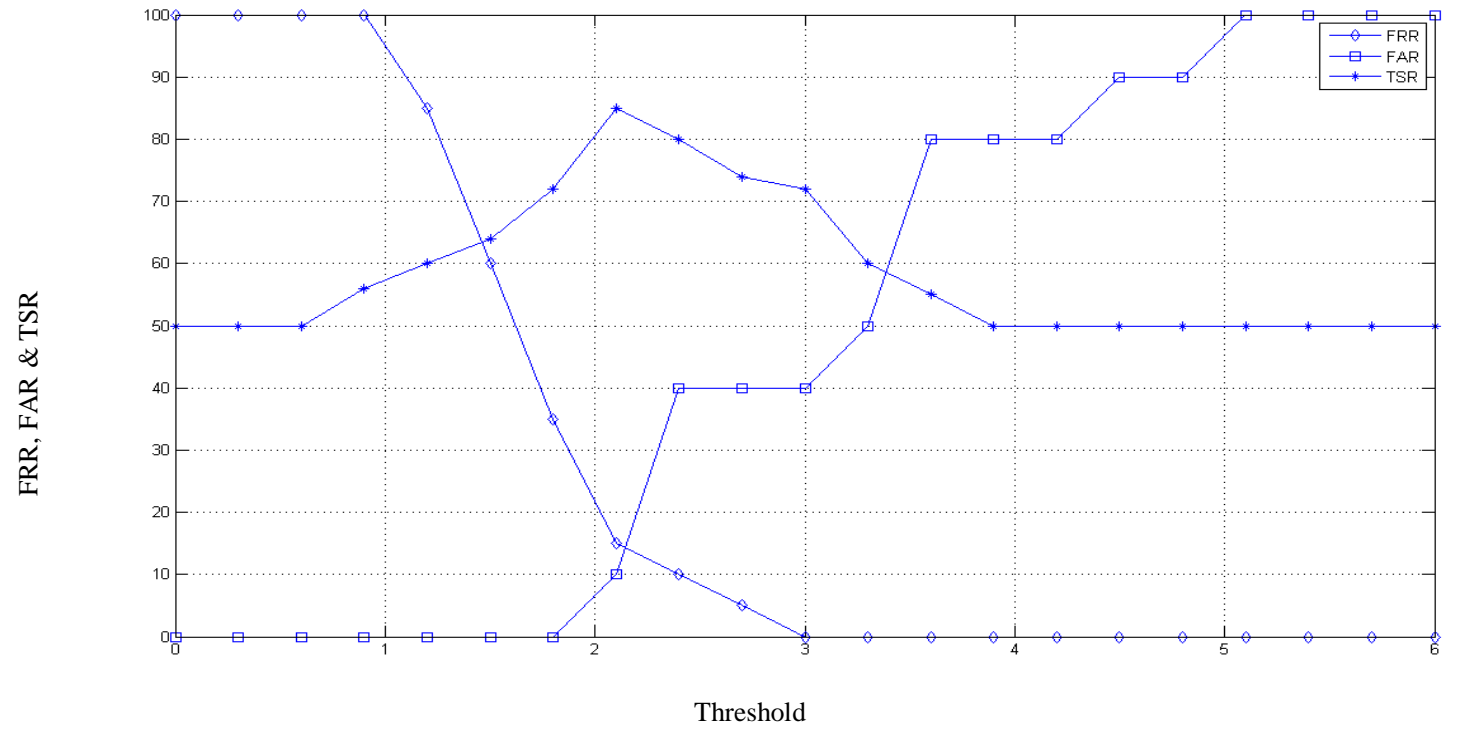

Figure 6. Variations of FRR, FAR and TSR with respect to different thresholds 
TABLE 3. VALUES OF FRR, FAR AND TSR FOR DIFFERENT THRESHOLDS

\begin{tabular}{|l|l|l|l|}
\hline Threshold & \multicolumn{1}{c|}{ FRR } & FAR & TSR \% \\
\hline 0 & 1 & 0 & 50 \\
\hline 0.3 & 1 & 0 & 50 \\
\hline 0.6 & 1 & 0 & 50 \\
\hline 0.9 & 1 & 0 & 50 \\
\hline 1.2 & 0.85 & 0 & 50 \\
\hline 1.5 & 0.6 & 0 & 60 \\
\hline 1.8 & 0.35 & 0 & 64 \\
\hline 2.1 & 0.15 & 0.1 & 72 \\
\hline 2.4 & 0.1 & 0.4 & 85 \\
\hline 2.7 & 0.05 & 0.4 & 80 \\
\hline 3 & 0 & 0.4 & 74 \\
\hline 3.3 & 0 & 0.5 & 72 \\
\hline 3.6 & 0 & 0.8 & 60 \\
\hline 3.9 & 0 & 0.8 & 55 \\
\hline 4.2 & 0 & 0.8 & 50 \\
\hline 4.3 & 0 & 0.9 & 50 \\
\hline 4.8 & 0 & 0.9 & 50 \\
\hline 5.1 & 0 & 1 & 50 \\
\hline 5.4 & 0 & 1 & 50 \\
\hline 5.7 & 0 & 1 & 50 \\
\hline 6 & 0 & 1 & 50 \\
\hline
\end{tabular}

\section{REFRENCES}

[1] B. N. Lavanya, K. B. Raja and K. R. Venugopal, "Fingerprint verification based on gabor filter enhancement," International Journal of Computer Science and Information Security,vol. 6, no. 2, pp. 138-144, 2009.

[2] S. Vasuki, L. Ganesan and R. Florin Raja Singh, "DT- WT based segmentation algorithm for color images," RTCSP Conference Proceedings, Coimbatore, pp. 207-212, 2009.

[3] Zhao Song and Liu Yuanpeng, "A novel image denosing scheme via combining dual tree complex wavelet transform and bandelets," IEEE International Symposium on Intellignet Information Technology Application, pp. 509-512, 2009.

[4] Chen Feng and Yu Song-nian, "Content - based image retrieval by DTCWT feature," IEEE International Conference on Computer Research and Development, vol. 4, pp. 283-286, 2011.

[5] Sathesh and Samuel Manoharan, "A dual tree complex wavelet transform construction and its application to image denosing," International Journal of Image Processing, Vol. 3, pp. 293-300, 2010.

[6] Lulian Voicu and Monica Borda, "New method of filters design for dual tree complex wavelet transform," IEEE International Symposium on Signals, Circuits and Systems, vol. 2, pp. 437-440, 2005.

[7] Mohd Shahid and Sumna Gupta, "Novel masks for multimodality image fusion using dtcwt," IEEE International Conference, TENCON, pp. 1-6, 2005.

[8] E. K. Yun and S. B. Cho, "Adaptive fingerprint image enhancement with fingerprint image quality analysis," International conference of Image and Vision Computing, pp. 101-110, 2006.

[9] M. P. Brankica and L. Maskovic, "Fingerprint minutiae filtering based on multiscale directional information," FACTA Universitatis-Series: Electronics and Energetics, vol. 20, pp.233-244, August 2007.

[10] F. A. Afsar, M. Arif and M. Hussain, "Fingerprint identification and verification system using minutiae matching," National Conference on Emerging Technologies, pp.141-146, 2004.

[11] G. Jagadeeswar Reddy, T. Jaya Chandra Prasad and M. N. Giri Prasad, "Fingerprint image denoising using curvelet transform," Proceeding of Asian Research Publishing Network Journal of Engineering and Applied Sciences, vol 3, no 3, pp. 31-35, June 2008.

[12] K. Zebbiche and F. Khelifi "Region-based watermarking of biometrics images: Case study in fingerprint images," Proceedings of International Journal of Digital Multimedia Broadcasting, pp. 1-13, 2008.
[13] Bhupesh Gour, T. K. Bandopadhyaya and Sudhir Sharma, "Fingerprint feature extraction using midpoint ridge contour method and neural network," Proceedings of International Journal of Computer Science and Network Security, vol.8, no.7, pp. 99-103, July 2008.

[14] Seung-Hoon Chae and Jong Ku Kim "Ridge-based fingerprint verification for enhanced security," Digest of Technical Papers International Conference on Consumer Electronics, pp 1-2, 2009.

[15] A. N. Marana, and A. K. Jain, "Ridge-based fingerprint matching using hough transform," IEEE Proceedings of the Brazilliab Symposium on Computer Graphica and Image Processing, pp. 112-119, October 2005.

[16] A. K. Jain, S. Prabhakar, and A. Chen, "Combining multiple matchers for a high security fingerprint verification system," Pattern Recognition Letters, Elsevier Science Direct, vol.20, pp 1371- 1379, 1999.

[17] Fanglin Chen and Jie Zhou, "Reconstructing orientation field from fingerprint minutiae to improve minutiae-matching accuracy," IEEE Transactions on image processing, vol. 18, no 4, pp 1665-1670, 2009.

[18] Chunxiao Ren and Yilong Yin, "A linear hybrid classifier for fingerprint segmentation," Fourth International Conference on Neural Computation, pp 33-37, 2008.

[19] Hartwig Fronthaler and Klaus Kollreider, "Local features for enhancement and minutiae extraction in fingerprints," IEEE Transactions on Image Processing, vol. 17, no 3, pp 354-363. 2008.

[20] D. R. Shashi Kumar, R. K. Chhotaray, K.B. Raja and Sabyasachi Pattanaik, "Fingerprint verification based on fusion of minutiae and ridges using strength factors," International Journal of Computer Applications, vol. 4, no.1, 2010.

[21] D. Maio, D. Maltoni, R. Cappelli, J.L. Wayman and A. K. Jain, "FVC2004: Third Fingerprint Verification Competition," Lecture Notes in computer science, pp.1-5, 2004.

[22] N.G. Kingsbury, "The dual-tree complex wavelet transform: A new technique for shift invariance and directional filters," Proceeding of 8th IEEE DSP Workshop, Utah, 1998.

[23] N.G. Kingsbury, "Image processing with complex wavelets," Philos. Trans. R. Soc. London A, Math. Phys. Sci., vol. 357, no. 1760, pp. 2543-2560, 1999.

[24] N.G. Kingsbury, "Complex wavelets for shift invariant analysis and filtering of signals," Appl. Comput. Harmon. Anal., vol. 10, pp. 234$253,2001$.

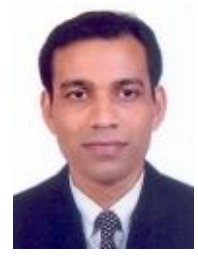

AUTHORS PROFILE

Jossy P. George currently serves as Assistant Professor of the Department of Computer Science at Christ University, Bangalore. He received the B. Sc. In Computer Science, Bachelor of Philosophy (B. Ph), Bachelor of Theology (B. Th) and Master of Computer Application (MCA). He has done his FDPM from IIM, Ahmedabad. He is pursuing his $\mathrm{Ph} . \mathrm{D}$. in Computer Science of Christ University under the guidance of Dr. K. B. Raja, Assistant Professor, Visvesvaraya College of Engineering. His research activities focus on algorithms for improved accuracy in fingerprint and iris biometrics.

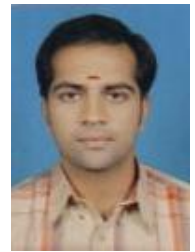

Abhilash S. K. obtained his BE in Electronics and Communication Engineering from DR Ambedkar Institute of Technology, Bangalore and is pursing his Postgraduate (ME) in Visvesvaraya college of Engineering, Bangalore. His research interests include Image processing and Biometrics.

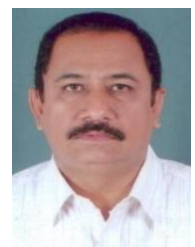

Dr. K B Raja is an Assistant Professor, Department of Electronics and Communication Engineering, University Visvesvaraya college of Engineering, Bangalore University, Bangalore. He obtained his $\mathrm{BE}$ and $\mathrm{ME}$ in Electronics and Communication Engineering from University Visvesvaraya College of Engineering, Bangalore. He was awarded Ph.D. in Computer Science and Engineering from Bangalore University. He has 85 research publications in refereed International Journals and Conference Proceedings. His research interests include Image Processing, Biometrics, VLSI Signal Processing, computer networks. 